\title{
EARLY DIAGNOSING AND TREATMENT \\ OF RHEUMATOID ARTHRITIS, BENEFITS \\ OF ANTI-CITRULLINATED PEPTIDES EXAMINATION
}

\author{
Belakova $\mathrm{G}^{1}$, Manka $\mathrm{V}^{1}$, ZaNOva $^{2}$, Racay $\mathrm{P}^{3}$. \\ ${ }^{1}$ Outpatient's Department and Center for Clinical Research in Rheumatology, Martin, Slovakia \\ ${ }^{2}$ National Institute of Rheumatic Diseases, Piestany, Slovakia \\ ${ }^{3}$ Comenius University and Jessenius Medical Faculty, Biomedical Centre Martin and Department of \\ Medical Biochemistry, Martin, Slovakia
}

\begin{abstract}
Background: Anti-citrullinated peptides antibodies (ACPA) are specific for rheumatoid arthritis and have been implicated in disease pathogenesis. ACPA examination is a new component of ACR/EULAR 2010 classification criteria for rheumatoid arthritis. ACPA positivity predicts a more erosive disease course with severe joint damage and extra-articular manifestations.

Objectives: To evaluate the benefits of ACPA examination in patients with early undifferentiated arthritis and patients with rheumatoid arthritis.

Methods: We examined patients with arthritis and tested them for ACPA positivity. In every individual patient we evaluated if ACPA examination was necessary to establish the diagnosis of rheumatoid arthritis, or to change treatment, or if the diagnosis could have been established without ACPA examination (ACR/EULAR 2010 classification criteria was met without ACPA scoring).

Results and Conclusions: We examined 833 patients with arthritis. There were 43 patients, or $62 \%$ of a subgroup of 69 who were ACPA positive whose ACPA examination was not needed - ACR/EULAR criteria was met without ACPA scoring. This number represents $5.1 \%$ of the total number examined. There were 15 patients, or $22 \%$ of the subgroup and $1.8 \%$ of the total whose diagnosis was revised to rheumatoid arthritis due to ACPA positivity - ACR/EULAR criteria was met solely with ACPA scoring. There were 11 patients (16\% and $1.3 \%$ ) whose medication was changed due to ACPA positivity. ACPA examination is useful in 3,1 \% of all examined patients. When we correlate data on ACPA positive patients, $38 \%$ of the patients profit from ACPA examinations. Considering the relatively low price of ACPA testing, this examination should not be excluded.
\end{abstract}

Key words: anti-citrullinated peptides antibodies, rheumatoid arthritis, ACR/EULAR 2010 classification criteria

\section{INTRODUCTION}

Rheumatoid arthritis is a complex disorder with many different aspects and many different forms at onset. It occurs in $1 \%$ of the population. It is characterised by chronic symmetric polyarthritis. Joint damage begins at the synovial membrane, and ongoing cartilage and bone destruction results in joint deformities.

A large number of patients also have extra-articular and systemic symptoms at the same time. Rheumatoid arthritis shortens life expectancy by approximately 10 years and it is a significant cause of disability and handicap in the population, ultimately leading to high economic loss in society (1).

Address for correspondence:

Gabriela Belakova, MD, Outpatient's Department and Center for Clinical Research in Rheumatology, Thurzova str. 15, Martin 03601 , Slovakia

email: gbelakova@gmail.com; phone 00421948039682 
Most of the recommended medications that are used to treat rheumatoid arthritis have an immunosuppressive effect and they may have multiple side effects and adverse events. Risk-benefit ratio should be assessed individually in every patient.

Like most of the systemic connective tissue diseases, rheumatoid arthritis has a multi-systemic heterogeneous clinical presentation. That is the reason why it may be difficult to establish a correct diagnosis in the early stages of the disease, and also why the "disease classification criteria" were formulated, consisting of clinical, histopathological and laboratory features. Establishing the correct diagnosis early and starting treatment adjusted to the individual patient as soon as the diagnosis is established is essential and a cardinal principle in order to prevent the patient's disability and handicap (2).

The key task is to identify markers with the highest possible sensitivity and specificity for rheumatoid arthritis (diagnostic markers) and also markers with the highest possible range of prediction of the severity of the disease (prognostic markers). Consequently, patients with a suspected severe course of the disease should be treated with more aggressive medication immediately after establishing diagnosis, and patients with a suspected mild course of the disease would be treated with less aggressive treatment to avoid a possible adverse event (3).

Anti-citrullinated peptides antibodies (ACPA) are autoantibodies that are highly specific for rheumatoid arthritis. Citrulline is a nonstandard amino acid created by deamination of the amino acid arginine by the enzyme PAD (peptidyl-arginine-deiminase). Citrullination is a common biological process that occurs during inflammation, apoptosis and cell differentiation. In the presence of certain genetic and environmental backgrounds, proteins are citrullinated excessively and they are not destroyed by the immune system, but contrariwise antibodies against citrullinated proteins are formatted and rheumatoid arthritis becomes manifested (4).

There are many ACPA tests available with different sensitivity and specificity for rheumatoid arthritis. ACPA examination is a new component of ACR/EULAR 2010 (American College of Rheumatology/European League against Rheumatism) classification criteria for rheumatoid arthritis. Low positivity of ACPA scores 2 points, while high positivity scores 3 of a possible 10 points from the individual score (4).

ACPA positivity predicts a more erosive disease course with severe joint damage and extraarticular manifestations. ACPA testing should be performed in individuals whose clinical signs suggest rheumatoid arthritis or who have already been diagnosed with undifferentiated arthritis. These antibodies also should be tested in patients who have already been diagnosed with rheumatoid arthritis to predict a possibly more progressive course of the disease (5).

\section{MATERIAL AND METHODS}

At our workplace (out-patient clinic) we performed a clinical study whose target was to evaluate the benefits of ACPA examination in patients with early arthritis and patients with rheumatoid arthritis. We examined patients with arthritis and tested them for ACPA positivity during a period of five years. All patients were educated and informed about our study, they agreed to participate and signed an informed consent form. In the group of patients with ACPA positivity we measured levels of antibodies. For low-level positivity we scored 2 points, for high-level positivity (three times higher than the upper limit of normal) we scored 3 points (according to ACR/EULAR 2010 classification criteria) of a possible 10 points of individual score.

We reviewed every individual patient and reconsidered whether ACPA examination was necessary to establish the diagnosis of rheumatoid arthritis or not. (Meaning the diagnosis could have been established without ACPA examination. The ACR/EULAR 2010 classification criteria was met without scoring ACPA positivity.) We also reviewed if the results of ACPA examination had led to changes in the patient's medication in the group of patients with an already established diagnosis of rheumatoid arthritis. 


\section{RESULTS}

In total we examined 833 patients with arthritis, of which 290 patients were diagnosed with rheumatoid arthritis according to ACR/EULAR 2010 classification criteria. There was a subgroup of 69 APCA positive patients, 63 with rheumatoid arthritis, 3 with osteoarthritis and 3 with systemic lupus erythematosus. Within this subgroup were 43 patients $(62 \%$ of the subgroup, $5.1 \%$ of the total) whose ACPA examination was not needed - ACR/EULAR 2010 criteria was met without ACPA scoring/ACPA positivity. There were 15 patients, (22\% of the subgroup, $1.8 \%$ of the total) whose diagnosis was revised to rheumatoid arthritis due to ACPA positivity - ACR/EULAR 2010 criteria was met solely with ACPA positivity scoring. There were 11 patients (16\%, of the subgroup, $1.3 \%$ of the total) whose medication was changed - augmented due to ACPA positivity or reduced due to ACPA negativity.

\section{DISCUSSION}

ACPA examination is recommended by ACR and EULAR as it is a part of the classification criteria for rheumatoid arthritis. Many studies have confirmed its diagnostic and prognostic values, but there had been no study performed to evaluate the importance of this examination for a single individual patient.

We discovered that ACPA examination is helpful and useful in $3.1 \%$ of all examined patients with arthritis (those patients whose diagnosis was revised or whose medication was changed due to ACPA examination results). When we correlate our data to the subgroup of ACPA positive patients we see that $38 \%$ of the patients profit from ACPA examinations.

The price of ACPA examination kits is relatively low, with prices varying in local laboratories. Average price for a single ACPA examination is approximately 5 USD / Euros. It is definitely worth the price, especially for patients who can avoid disability and functional handicap, their families, communities and society as a whole.

\section{CONCLUSIONS}

Rheumatoid arthritis is a chronic systemic disease that is shortening life-expectancy and possibly leading to functional disability and handicap. Since the disease has heterogeneous clinical manifestation we use ACR/EULAR 2010 classification criteria to establish the diagnosis. There are diagnostic and prognostic markers of the disease, one of them is anti-citrullinated peptides antibodies. Patients with a suspected severe course of the disease should be treated immediately after establishing diagnosis with more aggressive drugs to avoid progression to joint deformities and disability. Patients with a suspected mild course of the disease should be treated with less aggressive drugs without serious adverse events.

\section{REFERENCES}

1. van Steenberger HW, Ajeganova S, Forslind K, Swensson B, van der Helm-van Mil AHM. The Effect of Rheumatoid Factor and Anticitrullinated Peptide Antibodies on Bone Erosions in Rheumatoid Arthritis. Ann Rheum Dis 2015; e3: 74.

2. Britsemmer K, Ursum J, Gerritsen M, van Tuyl L, van Schaardenburg D. Validation of the 2010 ACR/EULAR Criteria for Rheumatoid Arthritis: Slight Improvement Over 1987 ACR Criteria. Ann Rheum Dis 2011; 70(8): 1468-70.

3. Smolen JS, Aletaha D, Bijlsma JW, Breedveld FC, Boumpas D, Burmester G, Combe B, Cutolo M, de Wit M, Dougados M, Emery P, Gibofsky A, Gomez-Reino JJ, Haraoui B, Kalden J, Keystone EC, Kvien TK, McInnes I, Martin-Mola E, Montecucco C, Schoels M, van der Hejde D. Treating Rheumatoid Arthritis to Target: Recommendations of an International Task Force. Ann Rheum Dis 2010; 69(4): 631-7. 


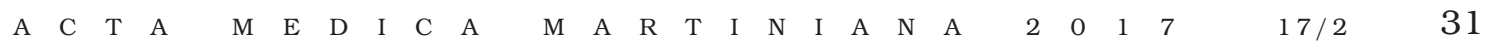

4. Kaneko Y, Kuwana M, Kameda H, Takeuchi T. Sensitivity and Specificity of 2010 Rheumatoid Arthritis Classification Criteria. Rheumatology(Oxford) 2011; 50(7): 1268-74.

5. Willemze A, Trouw LA, Toes RE, Huizinga TW. The Influence of ACPA Status and Characteristics on the Course of RA. Nat Rev Rheumatol 2012; 8(3): 144-52.

Received: March, 27, 2017

Accepted: April, 20, 2017 